\title{
Clinical validity of our color-imaging histo-diagnosis for
} cancer

\author{
Akio Sugitachi* ${ }^{* 1}$, Toshimoto Kimura ${ }^{1}$, Koki Otsuka ${ }^{1}$, Masanori Hakozaki ${ }^{1}$, Mizunori Yaegashi ${ }^{1}, M$ gegumu Kamishima ${ }^{1}$, Kohei Kume ${ }^{1}$, \\ Satoshi Nishizuka ${ }^{1}$, Noriyuki Uesugi ${ }^{2}$, Tamotsu Sugai ${ }^{2}$ and Akira Sasaki ${ }^{1}$ \\ ${ }^{1}$ Department of Surgery Iwate Medical University, Morioka 020-8505, Japan \\ ${ }^{2}$ Department of Molecular Diagnostic Pathology, Iwate Medical University, Morioka 020-8505, Japan
}

\begin{abstract}
We devised a color-imaging diagnosis for cancer, photodynamic histo-diagnosis (PDHD), with which we could clearly discriminate between cancer lesions and normal tissues. In an attempt to check the accuracy, we reexamined the PDHD method using two different types of colon tumors surgically harvested. The two specimens were treated according to our own procedures to microscopically observe reddish-pink fluorescence specifically emitted at cancer lesions. After which, such samples were modified to routine hematoxylin and eosin (HE) sections. Normal portion of the colon served as the control. To evaluate the diagnostic accuracy, we compared our PDHD findings with that of the HE sections. The two colonic samples emitted reddish-pink fluorescence by the PDHD method, which suggested that they were cancer lesions. While, diagnosis with the HE sections revealed one was "cancer in adenoma" and the other was "adenoma with no signs of cancer". This result suggested all was not cancer that emitted reddish fluorescence with our PDHD.
\end{abstract}

\section{Introduction}

Clinical photodynamic diagnosis (PDD) and photodynamic therapy (PDT) have been often carried out in urological fields and neuro surgeries [1-4]. In PDD and PDT, aminolevulinic acid (ALA) is mainly used as a photosensitizer [5]. Based on the ALA-PDD theory, we took its rationale into our microscopic diagnostic manner as photodynamic histo-diagnosis (PDHD) and photodynamic cyto-diagnosis (PDCD) [6]. Using the novel method, we could visually discriminate a cancer cell from pathologically uncertain specimens in our previous study [6]. In this paper, we described further results of the PDHD to reconsider on its accuracy comparing with routine method with HE sections.

\section{Materials and methods}

Materials and methods have been already published [6]. Two different types of tumors which had grown at the colon of one patient were subjected to clinical specimens. After colectomy, each lesion was immediately treated for PDHD to detect the fluorescence. Then, the same samples were transformed to routine HE sections. We microscopically compared the PDHD findings with that of HE sections to evaluate diagnostic accuracy of our own PDHD. The HE sections were examined by one pathologist who was blinded to the PDHD.

\section{Results}

The two specimens treated with the PDHD method emitted reddish-pink fluorescence, by which we interpreted them as cancer. The normal sample showed no fluorescence (Figure 1). The HE sections once treated with PDHD method also radiated reddish fluorescence under blue light. In white light mode, they revealed one was "carcinoma in adenoma" and the other was "adenoma with no signs of cancer" (Figures 2, 3 and 4).

\section{Discussion}

Using PDHD method, we could visually discriminate cancer from normal tissue by identifying reddish-pink fluorescence. In the previous study, we could also diagnose bile tract cancer by our own PDCD [6]. While in this study, we could not distinguish an adenoma

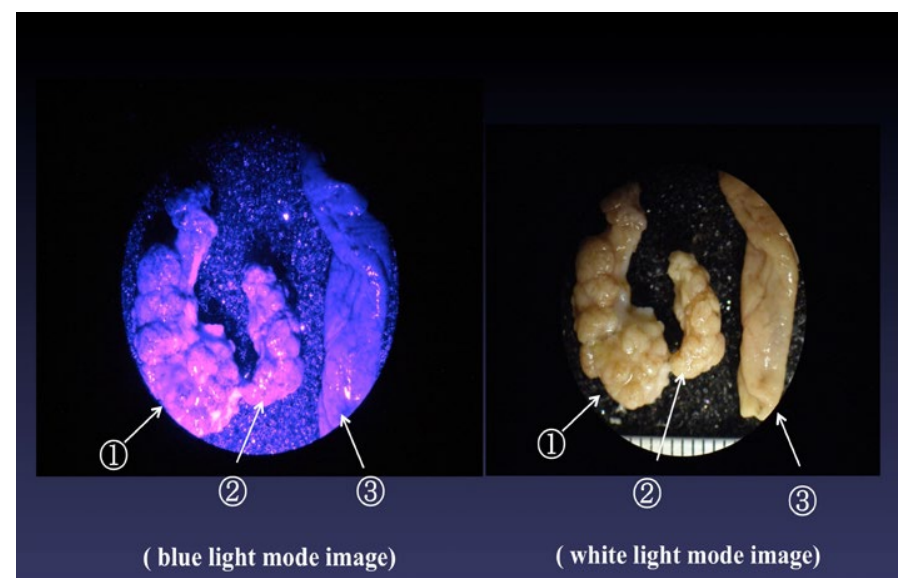

Figure 1. Three magnified colonic specimens harvested from one patient. Specimens (1) and (2) emitted reddish-pink fluorescence under the blue light (wave length $380-410 \mathrm{~nm}$ ) and Specimen (3) radiated no fluorescence. The three specimens emitted no fluorescence under white light mode.

Correspondence to: Akio Sugitachi, MD, PhD, Department of Surgery, Iwate Medical University, 1-19, Uchimaru, Morioka 020-8505, Japan, Tel: 019-6515111; Fax: 019-651-7166; E-mail: akiosugi@iwate-med.ac.jp

Key words: cancer, PDHD, reddish pink fluorescence, HE section

Received: October 15, 2015; Accepted: October 27, 2015; Published: October 30,2015 


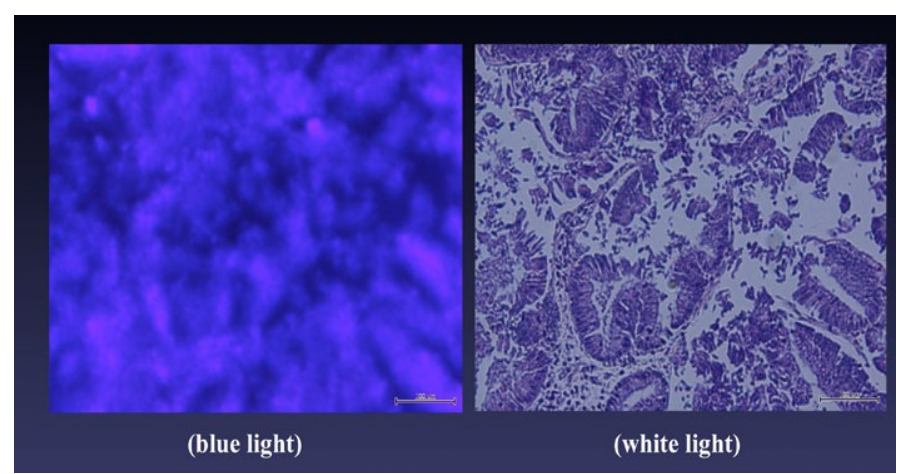

Figure 2. Microscopic findings of the specimen (1) (HE section). The sample emitted reddish fluorescence under the blue light, while the cellular margins were obscure comparing with that of in white light. In white light mode, well differentiated adenocarcinoma with partial adenoma formation was observed.

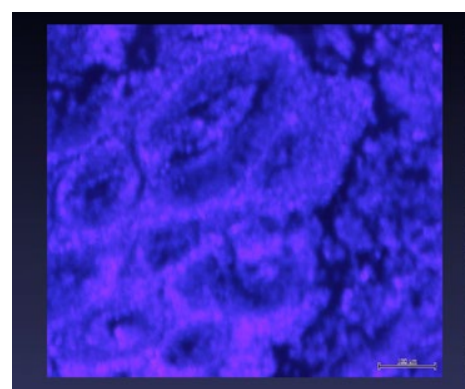

(blue light)

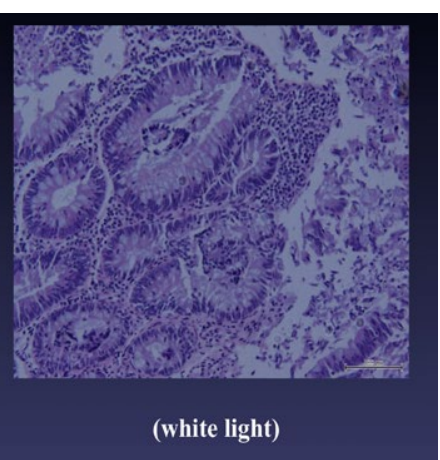

Figure 3. Microscopic findings of the specimen (2) (HE section). The sample also radiated reddish fluorescence in the blue light. The whole image was clearer than that of shown in Figure 2. In white light mode, moderately differentiated tubular adenoma formation with no malignant changes was seen.

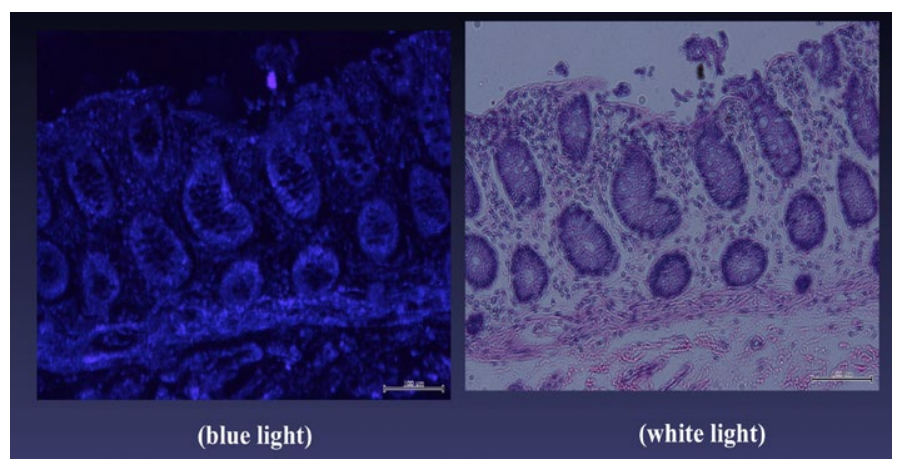

Figure 4. Microscopic findings of the specimen (3) (HE section). No reddish fluorescence appeared in the blue light mode. Normal mucosa of the colon with no neoplastic findings were observed in white light mode.

from a carcinoma, since the two specimens similarly showed reddish fluorescence in blue light mode. Our PDHD unexpectedly provided a false-positive finding this time. The fact strongly suggested that the specimen emitted reddish-pink fluorescence by our PDHD method not always represented cancer lesion. We have learned luminescence of the reddish fluorescence appeared in our PDHD had no relation to the grade of malignancy of the tumors. Therefore, we considered since intracellular accumulation of color-imaging agents, such as PpIX in ALA-PDD and ${ }^{18} \mathrm{~F}$ - fluorodeoxyglucose (18F-FDG) in PET/CP [7] were enhanced in the areas where the cell proliferation and cell growth metabolism were vigorous $[7,8]$, such fluorescence would tend to strongly appear in neoplasm, unrelated to be malignant or benign. We supposed the false-positive finding this time was mainly due to such a reason.

Microscopically, photodynamic efficacy of the fluorescence seemed to be also correlated with intracellular localization of the PpIX. Sailer and others reported that PpIX was predominantly distributed in lipophilic environment [9]. In our present study, we could also observe the fluorescence of PpIX in the HE sections which had once treated for PDHD. While the fluorescence was too weak to clearly distinguish its intracellular distribution. Since our specimens were simply immersed into ALA-containing solution for the ALA-PDHD preparation, the ALA-induced PpIX might be limitedly synthetized only on surface of the cells, resulting the intracellular PpIX distribution could not be clearly recognized. The intensity of fluorescence induced by our PDHD gradually decreased due to photo-bleaching to maintain it for about 10 days in a dark box. NPG (n-propyl gallate) was reported as a useful enhancer of PpIX [10]. Although we have never tried it yet it will be promising to improve the PDD-efficiency.

In clinical cases, false-positive findings happened due to tangent effects during PDD operation [11]. They accordingly applied a new flexible PDD instrument to resolve the problem. Since the PpIX radiates red fluorescence by blue light irradiation [5], the angle of the excitation beam onto the target will be an important factor to obtain suitable fluorescence intensity. In our PDHD and PDCD, a rightangled blue light application to the sample was also necessary. We adequately adjusted the angle by our own hand-working.

Recently, clinical PDD and/or PDT apply highly efficient devices and optical probes such as a confocal laser endoscope and an endomicroscope, etc. They will diagnostically and therapeutically bring a considerable favor. While, detail reports concerning the clinical effectiveness and cost performance owing to such multi-functional instruments are still limited.

As for the advantage of our PDHD and PDCD, materials and tools necessary for the purpose were simple and inexpensive. Nevertheless they could microscopically provide color images of the targets. To maintain diagnostic accuracy of our PDHD and PDCD, further statistical analyses on our clinical PDHD and PDCD data will be required.

\section{Acknowledgements}

The authors are grateful to Mr. Naoki Omura, Iwate Medical University, Morioka, Japan for technical assistance in the experiments.

\section{References}

1. Mowatt G, N'Dow J, Vale L, Nabi G, Boachie C, et al. (2011) Photodynamic diagnosis of bladder cancer compared with white light cystoscopy: Systematic review and metaanalysis. Int J Technol Assess Health Care 27: 3-10. [Crossref]

2. Cordeiro ER, Anastasiadis A, Bus MT, Alivizatos G, de la Rosette JJ, et al. (2013) Is photodynamic diagnosis ready for introduction in urological clinical practice? Expert Rev Anticancer Ther 13: 669-680. [Crossref]

3. Friesen SA, Hjortland GO, Madsen SJ, Hirschberg H, Engebraten O, et al. (2002) 5-Aminolevulinic acid-based photodynamic detection and therapy of brain tumors (review). Int J Oncol 21: 577-582. [Crossref]

4. Grieb P (2004) 5-Aminolevulinic acid (ALA) and its applications in neurosurgery Neurol Neurochir Pol 38: 201-207. [Crossref]

5. Campbell DL, Gudgin-Dickson EF, Forkert PG, Pottier RH, Kennedy JC (1996) Detection of early stages of carcinogenesis in adenomas of murine lung by 5-aminolevulinic acid-induced protoporphyrin IX fluorescence. Photochem Photobiol 64: 676-682. [Crossref] 
6. Sugitachi A, Otsuka K, Kimura T, Hakozaki M, Yaegashi M, et al. (2015) Colorimaging histodiagnostic approach for cancer. Integr Mol Med 2: 231-233.

7. Hwang JP1, Woo SK, Yoon SY, Jeong SY (2015) The potential usefulness of (18) F-FDG PET/CT for detecting colorectal carcinoma and adenoma in asymptomatic adults. Ann Nucl Med 29: 157-163. [Crossref]

8. Endlicher E, Gelbmann CM, Knüchel R, Fürst A, Szeimies RM, et al. (2004) Hexaminolevulinate-induced fluorescence endoscopy in patients with rectal adenoma and cancer: a pilot study. Gastrointest Endosc 60: 449-454. [Crossref]

9. Sailer R, Strauss WS, Wagner M, Emmert H and Schneckenburger H (2007) Relation between intracellular location and photodynamic efficacy of 5-aminolevulinic acidinduced protoporphyrin IX in vitro. Comparison between human glioblastoma cells and other cancer cell lines. Photochem Photobiol Sci 6: 145-151. [Crossref]

10. Hirano T, Hagiya Y, Fukuhara H, Inoue K, Shuin T, et al. (2013) Improvement of aminolevulinic acid (ALA)-mediated photodynamic diagnosis using n-propyl gallate. Photodiagnosis Photodyn Ther 10: 28-32. [Crossref]

11. Fukuhara H, Kureishi M, Khoda T, Inoue K, Tanaka T, et al. (2015) The Utility of Flexible Fluorescence-Cystoscope with a Twin Mode Monitor for the 5-Aminolevulinic Acid-Mediated Photodynamic Diagnosis of Bladder Cancer. PLoS One 10: e 0136416. [Crossref]

Copyright: $(02015$ Sugitachi A. This is an open-access article distributed under the terms of the Creative Commons Attribution License, which permits unrestricted use, distribution, and reproduction in any medium, provided the original author and source are credited. 